\title{
ASSESSING THE FEASIBILITY OF UAV-BASED LIDAR FOR HIGH RESOLUTION FOREST CHANGE DETECTION
}

\author{
L. O. Wallace *, A. Lucieer and C. S. Watson \\ Survey and Spatial Science Group \\ School of Geography and Environmental Studies \\ University of Tasmania \\ Hobart, Tasmania, Australia \\ Luke.Wallace@utas.edu.au
}

KEY WORDS: Unmanned Aerial Systems, LiDAR, Forestry, Change Detection

\begin{abstract}
:
Airborne LiDAR data has become an important tool for both the scientific and industry based investigation of forest structure. The uses of discrete return observations have now reached a maturity level such that the operational use of this data is becoming increasingly common. However, due to the cost of data collection, temporal studies into forest change are often not feasible or completed at infrequent and at uneven intervals. To achieve high resolution temporal LiDAR surveys, this study has developed a micro-Unmanned Aerial Vehicle (UAV) equipped with a discrete return 4-layer LiDAR device and miniaturised positioning sensors. This UAV has been designed to be low-cost and to achieve maximum flying time. In order to achieve these objectives and overcome the accuracy restrictions presented by miniaturised sensors a novel processing strategy based on a Kalman smoother algorithm has been developed. This strategy includes the use of the structure from motion algorithm in estimating camera orientation, which is then used to restrain IMU drift. The feasibility of such a platform for monitoring forest change is shown by demonstrating that the pointing accuracy of this UAV LiDAR device is within the accuracy requirements set out by the Australian Intergovernmental Committee on Surveying and Mapping (ICSM) standards.
\end{abstract}

\section{INTRODUCTION}

Airborne Light Detecting and Ranging (LiDAR) data has become an increasingly important tool for the scientific investigation of forest structure. The use of discrete return observations have reached a level of maturity which allows the accurate generation of forest resource inventories for operational use to become increasingly common (Woods et al., 2011). Limited multi-temporal studies using LiDAR data have also shown potential for assessing forest dynamics such as changes in biomass, gap fractions (Vepakomma et al., 2008) as well as for assessing the uncertainty in growth prediction models (Hopkinson, 2008). The use of LiDAR data for multi-temporal forest surveys are, however, restricted by the high cost of data collection as well as restrictions on flying seasons in some areas. Data collected from repeat surveys are further complicated by the often large time periods between surveys. As such these surveys are often performed using different sensor sets at different flying conditions. For example, Vepakomma et al. (2008) used data from surveys with two different sensors flown at different altitudes $(700 \mathrm{~m}$ and 1000 $\mathrm{m})$. It has been shown that these datasets are not directly comparable as different instruments produce data with different properties (Næsset, 2009). These restrictions make the assessment of forest change using full scale airborne data difficult, restricting scientific investigation and making practical use for forestry management infeasible.

Mini-Unmanned Aerial Vehicles (UAV) are a platform which can be used within targeted very high spatial and temporal resolution surveys at a low cost. The use of these systems in combination with miniaturised laser scanners has been highlighted as a platform which will allow high frequency multi-temporal forest studies to be performed with the same instrumentation set (Jaakkola et al., 2010). The UAV-borne LiDAR system outlined in Jaakkola et al. (2010), for example, highlights the key advantages of UAVs. However, the effect of flight conditions for measuring forest metrics is yet to be thoroughly examined. The use of mini-UAVs suggest that survey conditions will typically include lower flying heights, higher point densities, and a smaller survey areas. Furthermore, the miniaturised laser scanner has been developed for applications other than mapping (automotive and robotics applications for example) and are not as highly developed as laser scanners used on-board full scale systems.

The effect of different flying conditions on the collection of discrete return LiDAR data within forest has been well studied (Lovell et al., 2005; Goodwin et al., 2006; Disney et al., 2010). Such studies have often been completed with the aim of determining optimum flying conditions for LiDAR surveys. These studies have shown that point density, beam divergence, scan angle as well as the internal properties of the scanner, such as the triggering mechanism all have an effect on the derivation of tree metrics and that these effects are more pronounced at finer measurement scales (i.e. the individual tree level). Lovell et al. (2005), for example, suggests that tree height measurements are improved in data sets with higher point density due to the increased likelihood that the tree top will be sampled. Furthermore, it has been shown that the use of large scan angles will result in a reduction in the number of lower canopy returns and effect canopy cover estimates (Morsdorf et al., 2006). The effect of survey conditions are important to consider for UAV-borne LiDAR surveys. These systems offer higher point densities and depending on the chosen survey conditions and area of interest may require the use of higher scan angles to capture an area of interest. Furthermore, the miniaturised sensors on these systems typically have a higher beam divergence, less sensitive diodes, different triggering mechanisms and the ability to utilise higher scanning angles (up to $180^{\circ}$ ). As such the optimum flying conditions, using these low-cost sensors mounted on UAVs, for repeatable determination of forest metrics from LiDAR data requires further investigation. This analysis can assume similar effects of flying height as shown in these prior studies to provide an initial hypothesis for determining the optimum conditions for performing UAV-borne surveys. 
The objectives of this paper are to assess the ability of UAV platforms for monitoring high resolution change within a Eucalyptus Nitens plantation. This will be achieved by examining the capabilities of a UAV-borne LiDAR system, carrying the same model scanner as described in (Jaakkola et al., 2010), to resolve plot level forest metrics and assess the repeatability of these metrics. The effects of the spatial accuracy of these systems, varying beam width, point density and scan angle will be assessed by altering the system's altitude and then manipulating the resultant point clouds to isolate the effects of each factor.

\section{METHODS}

\subsection{Hardware}

The capabilities of the TerraLuma UAV-borne LiDAR system developed at the University of Tasmania will be assessed in this study (shown in Figure 1). The platform consists of a multirotor UAV (OktoKopter Droidworx/Mikrokopter AD-8) which is used to carry the sensor payload. This particular system is capable of carrying up to $2.8 \mathrm{~kg}$ for a duration of 3- 4 min which is sufficient time to cover a plot sized area within $100 \mathrm{~m}$ of the take off point. The system is equipped with an on-board autopilot allowing predefined flight paths to be followed which ensure an efficient use of this flight time.

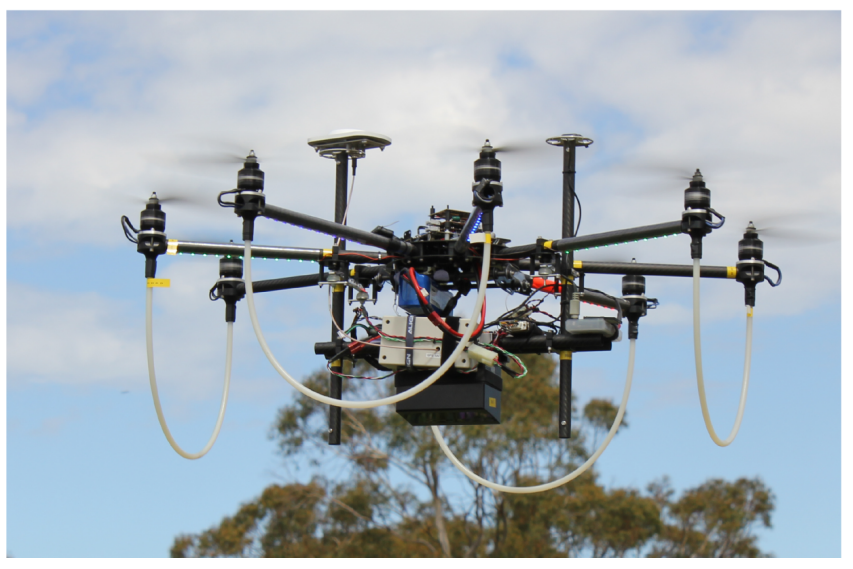

Figure 1. The multi-rotor UAV platform used within this study. The laser scanner on-board this system is an Ibeo LUX automotive scanner.

The sensor payload is vibration isolated from the main platform through 4 silicon mounts. This payload consists of a position and orientation system (POS), a laser scanner and a data logging pc. The POS consists of a MEMs based IMU, a dual frequency GPS receiver and an HD Video camera. The high rate measurements from the IMU are fused with observations of position and velocity from the GPS and orientation from the camera to ensure high accuracy observations of position and orientation are made for use in the generation of a point cloud (as outlined in Wallace et al. (2011)). This payload is stand-alone from the sensors used in the auto-pilot and all processing is performed offline. The on-board laser scanner is an Ibeo LUX automotive sensor which measures points in four scanning layers and in doing so can record up to 22000 returns/s. The scanner has a measurement range of up to $200 \mathrm{~m}$ with a repeatability of $0.10 \mathrm{~m}$. The beam divergence of the Ibeo LUX laser scanner is $0.08^{\circ}$ across track and $1.6^{\circ}$ along track. The Ibeo LUX can record up to 3 returns per pulse and records a pulse length measurement for each return.

\begin{tabular}{ccccc}
\hline Flight & 1 & 2 & 3 & 4 \\
\hline Altitude $(\mathrm{m})$ & 30 & 50 & 70 & 90 \\
points $/ \mathrm{m}$ & 77 & 45 & 19 & 10 \\
Footprint Diameter & 0.83 & 1.30 & 1.95 & 2.38 \\
across(along) (m) & $(0.04)$ & $(0.07)$ & $(0.10)$ & $(0.12)$ \\
Maximum Scan & 29.75 & 21.75 & 20.5 & 9.25 \\
Angle $\left({ }^{\circ}\right)$ & & & &
\end{tabular}

Table 1. Flight conditions over the field measured pre-pruning assessment plot.

\subsection{Study Area and Data Collection}

A 5 year old Eucalyptus Nitens plantation coup was chosen as the study area. The coup is located near the town of Franklin in Tasmania, Australia (Figure 2). The area has a mean elevation of $450 \mathrm{~m}$ and consists of terrain with a $20^{\circ}$ east facing slope. The trees stand approximately $10 \mathrm{~m}$ tall and were due to be pruned within 2 months of the LiDAR survey.

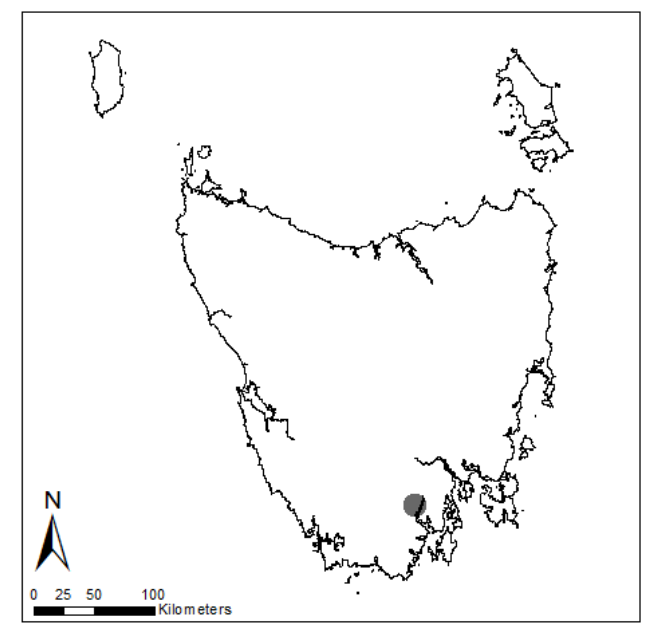

Figure 2. The state of Tasmania with a dot showing the location of the study area.

UAV-borne LiDAR data was collected from four flights following a $120 \mathrm{~m}$ transect in both forward and reverse directions. In each flight this transect was flown in both forward and reverse directions. Each flight was flown with an average velocity of $4.0 \mathrm{~m} / \mathrm{s}$ at a constant height above the take-off point. Four $12.62 \mathrm{~m}$ radius circular plots were extracted from each of the point clouds for use in this analysis. One of these areas is located over a future pre-pruning assessment plot. This ground inventory will be used in future analysis of the collected data. The properties of the data acquisition are given in Table 1 based on the above ground flying height over this ground inventory plot. The slope of the terrain and constant flying height above take-off allowed a variety of flying heights to be assessed over the remaining plots. These four flying heights show the significant variation in footprint diameter, point density and scan angle of the UAV system. The footprint diameter at $30 \mathrm{~m}$ can be considered similar to some modern full scale data as used in (Yu et al., 2011) for example. However, all other data is collected with a significantly larger footprint. The lowest flying height of $30 \mathrm{~m}$ ensured safe operating distance of approximately $10 \mathrm{~m}$ above the trees the top of the slope.

Generating point clouds separately for the forward and reverse transects allowed comparison both at the various flying height, as 
well as, at similar flying heights. This resulted in a set of eight point clouds at four different flying heights for each of the five plots. Each of the generated point clouds were filtered to produce ground and non-ground points using the methods outlined in (Streutker and Glenn, 2006). The ground points were then used to create a bare earth digital terrain model (DTM). The heights of all non-ground points above this surface were determined by subtracting the DTM value at the corresponding location. Points with an Above Ground Height (AGH) of more than $0.5 \mathrm{~m}$ were considered as vegetation and used in the derivation of forest metrics.

Finally, the high density point clouds were then decimated to lower point densities in steps of 10 point per $\mathrm{m}$ through to a smallest density of 0.5 points per $\mathrm{m}$. Several decimation methods were trialled. However, the process which produced the most visibly similar point clouds to those produce at the higher flight altitudes involved creating a grid with a randomly selected starting point and a cell size chosen to match the targeted point density. For each cell within the grid the first return closest to the center of the grid was added to the decimated point cloud. Any related second and third returns were also included in the decimated point cloud.

\subsection{Assessment of Survey Conditions}

In order to assess the ability of the UAV system to derive forest metrics under different flying conditions, a set of descriptive statistics were generated for each point cloud. The statistics used were chosen as they have been shown to represent to key canopy attributes and can be derived directly from the point cloud. The set of statistics includes AGH quantiles, mean AGH, standard deviation, skewness, kurtosis and coefficient of variation which have been shown to provide an estimate for foliage, canopy structure and the distribution of biomass within the canopy (Lim and Treitz, 2004; Donoghue et al., 2007). Furthermore, the proportion of canopy hits to total hits provides an accurate representation of canopy cover (Morsdorf et al., 2006). These statistics were calculated for only first returns and for all non-ground returns above $1 \mathrm{~m}$ falling within the plot boundaries and for each cell within a $1.0 \mathrm{~m}$ grid covering the plot area. Statistics were only generated for cells with fifteen or more above ground points falling within that cell boundary.

The point cloud and statistic sets were then qualitative and quantitative compared for each of the generated flying heights. Initially, all non-decimated point clouds were compared in order to determine the overall effect of flying height of the UAV on the measurement of forest structure. Following this the decimated point clouds were used in the comparisons with the aim of separating out the individual variations due to point density, scan angle and footprint size. Point density was separated from the other effects by comparing decimated point clouds from the same flight. This approach isolated point density from all other survey factors. This was more difficult to achieve for determining the effect of scan angle and footprint size. The effect of scan angle was analysed by comparing the gridded statistics of point clouds decimated to the same density. This approach allowed cells with up to $20^{\circ}$ differences in scan angle to be compared. Due to difference in range the footprint size was still likely to be contributing to any differences found. A similar approach was taken for footprint size, however, only gridded areas with a scan angle difference of less than $3^{\circ}$ were included in the analysis.

The accuracy of the horizontal measurement of tree locations was also examined. The approach used involved comparing the manually derived location of 50 trees randomly selected from within the plot areas. This location was found by manually selecting a set of above ground points which represented a single tree crown. The location of a tree was then defined as the highest point within this group of returns.

\section{RESULTS}

\subsection{Flying Height}

Figure 3 shows a point cloud of a plot generated at a flight altitude of $50 \mathrm{~m}$. Visually the structure of an individual tree can be seen in the point cloud. Furthermore, there are a sufficient number of returns from the ground in order to derive a digital terrain model for use in the determination of vegetation AGH.

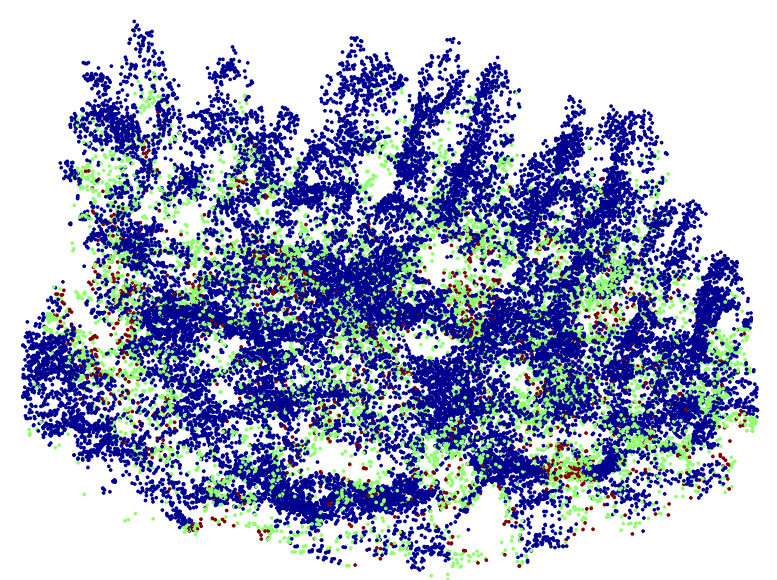

Figure 3. An example point cloud of a single plot generated at an average altitude of $48.32 \mathrm{~m}$. First (blue), second (green) and third (red) returns are all shown in the figure.

The distribution of the AGH of first and last vegetation returns was found to be highly affected by the altitude of the UAV platform. This is demonstrated in Figure 4 for a single plot, with similar patterns observed across the remain four plots. The variation with altitude of the return distributions is similar for both first and last returns. The distribution of points was similar for all point clouds generated from flights below $50 \mathrm{~m}$. Within these point clouds a significant number of first returns are from the under-story and lower canopy with a secondary group of returns in the range $5-10 \mathrm{~m}$ above the ground representing the upper canopy. For point clouds generated from flights greater than 50 $\mathrm{m}$, there is a significant attenuation in the number of returns between $5-10 \mathrm{~m}$. With the number of vegetation returns in this range dropping to almost zero in a flight at $90 \mathrm{~m}$.

This decrease in upper canopy returns also causes significant variation in the statistics calculated. For instance, there is less than $7 \%$ variation seen in AGH quantiles for all point clouds captured at less than $50 \mathrm{~m}$. However, this increases up to $120 \%$ for differences between these point clouds and the 70 and $90 \mathrm{~m}$ point clouds. This difference is demonstrated in Figure 5, which shows that repeatable quantile values are derived for flights of similar heights below $50 \mathrm{~m}$. the comparison of point clouds from forward and reverse transects found variations of no greater than $3 \%$ in the calculation of plot level AGH quantiles.

A similar pattern was found for the remaining statistics. The statistics which explain canopy structure (skewness, kurtosis, percentile differences, proportion of canopy hit) are all stable at the plot level for the point clouds captured at lower altitudes between 20 and $50 \mathrm{~m}$. For point clouds captured above a height 


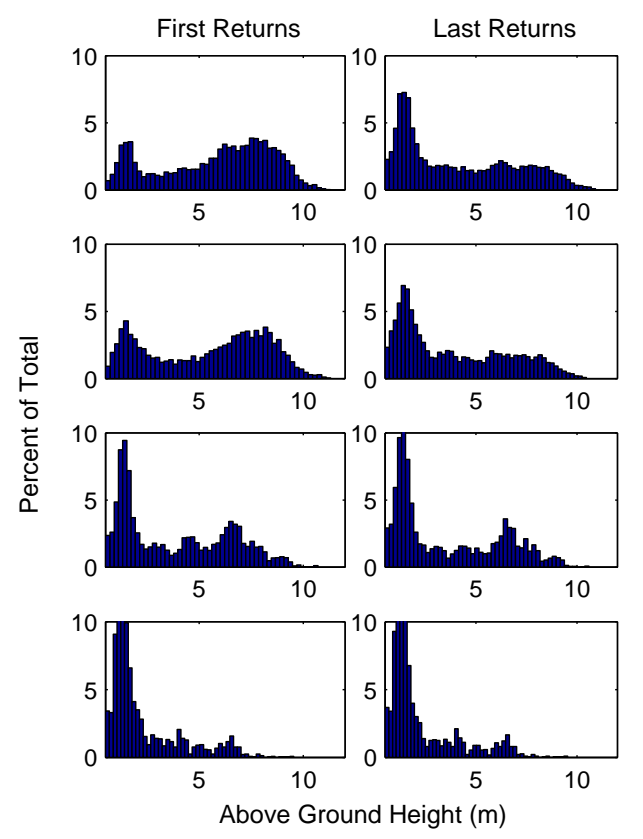

Flying Height

$30 \mathrm{~m}$

$70 \mathrm{~m}$

$90 \mathrm{~m}$

Figure 4. Histograms of the above ground height of vegetation returns over a single plot for point clouds captured at above ground flying heights of a) $30 \mathrm{~m}$, b) $50 \mathrm{~m}$, c) $70 \mathrm{~m}$ and d) $90 \mathrm{~m}$. There is an obvious attenuation of upper canopy returns due to flight altitude.

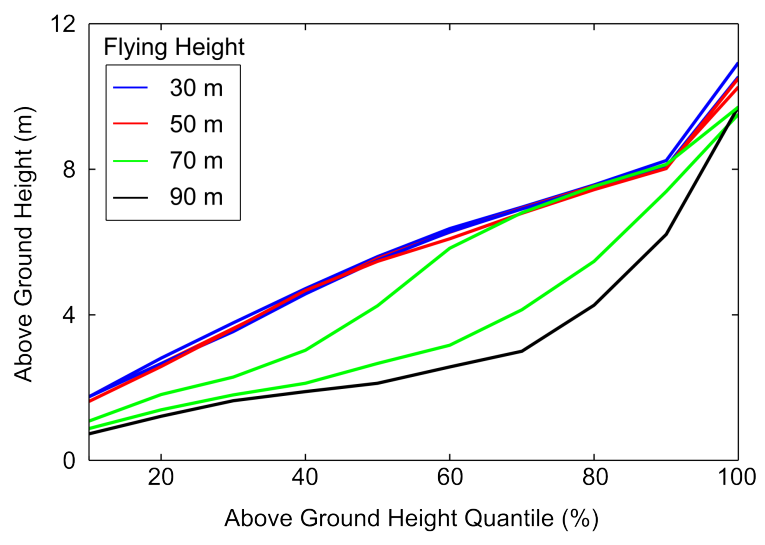

Figure 5. Above ground height quantiles measured from various flying heights for a single plot.

of $50 \mathrm{~m}$ these statistics vary by more than $100 \%$. This variation in the point clouds captured at greater altitudes suggests that these point clouds are not capturing the true canopy structure. As such point clouds from flights with an altitude of more than $50 \mathrm{~m}$ above the ground will not be used in any further analysis.

\subsection{Horizontal Accuracy}

The location of 50 random trees as found in the measurements of point clouds below $50 \mathrm{~m}$ were compared. This resulted in a minimum of four tree locations being observed for each tree. The mean repeatability of an individual tree location $0.36 \mathrm{~m}$ with a standard deviation of $0.24 \mathrm{~m}$. The maximum difference between any two tree locations of $0.96 \mathrm{~m}$ was found between two flights at $50 \mathrm{~m}$ at a scan angle of approximately $20^{\circ}$ in both flights. This suggests that accuracy is highly affected by increased scan angles and flying heights. The sampling densities at flying heights below
$50 \mathrm{~m}$ suggests the top of a tree is likely to be sampled and any error is likely to be due to errors in the position, orientation or calibration of the LiDAR system.

\subsection{Point Density}

The effect of point density on the calculation of quantiles at the plot level is significantly different to that seen due to the variation of different flying heights. Decreasing point densities primarily result in a small decrease in the number of points measured at the very top of the crown. This is illustrated by a decrease of up to $1.2 \mathrm{~m}$ in the 90 th and 100 th percentiles (when comparing 10 points per $m$ to 77 points per $m$ ). As the positively skewed canopy height distribution tends toward symmetry there is also an increase of mid-canopy height quantiles. A reduction in point density from 77 points per $m$ to above 30 points per $m$ (as shown in Figure 6) was found to have no significant effect on any of the plot level statistics calculated.
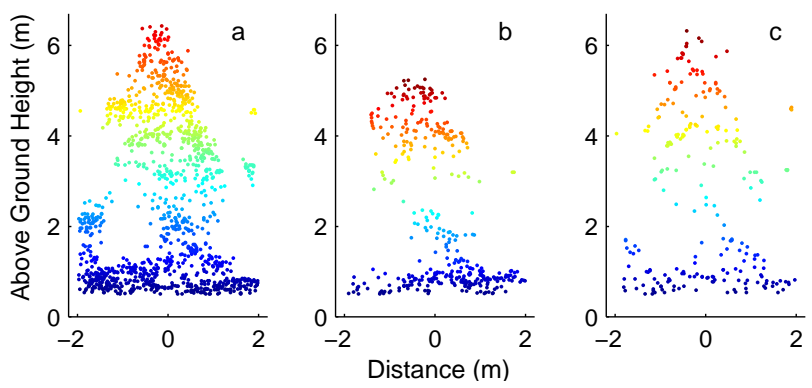

Figure 6. The effect of point density at the level of an individual tree. Showing a) full density point cloud at $30 \mathrm{~m}, \mathrm{~b}$ ) full density point cloud at $70 \mathrm{~m}$ and c) decimated point cloud at $30 \mathrm{~m}$

At the level of an individual grid cell more variation due to to point density is seen in the calculation of some of the statistics. This is primarily due to an under sampling of the canopy at this point density. For point densities below 40 points per $\mathrm{m}$ there is a significant reduction of up to $10 \%$ in the upper level canopy density metrics. Furthermore, the percentage of canopy returns vary by up to $50 \%$.

\subsection{Scan Angle}

The primary effect of increased scan angle is a shadowing on the far side of the trees (as demonstrated Figure 7). The statistics for grid cells in these shadows were not calculated due to the lack of information. Therefore, the comparison of most statistics from cells with a sufficient number of returns at different scan angles, showed only a slight increase in variance for cells with large scan angle differences.

\subsection{Footprint Size}

There was no significant variation found due to footprint sizes (range of $0.6-1.3 \mathrm{~m}$ ) in flights lower than $50 \mathrm{~m}$. As this analysis was restricted due to the necessity of scan angle similarity there may exist greater variation towards the edges of the plots due to increased ranges of footprint sizes.

\section{DISCUSSION AND CONCLUSIONS}

The results of this study suggest that a repeatable set of statistics can be derived from multiple flights of a UAV-borne LiDAR system. To achieve this, however, the flying height of the system needs to be restricted to less than $50 \mathrm{~m}$. The primary effect of 


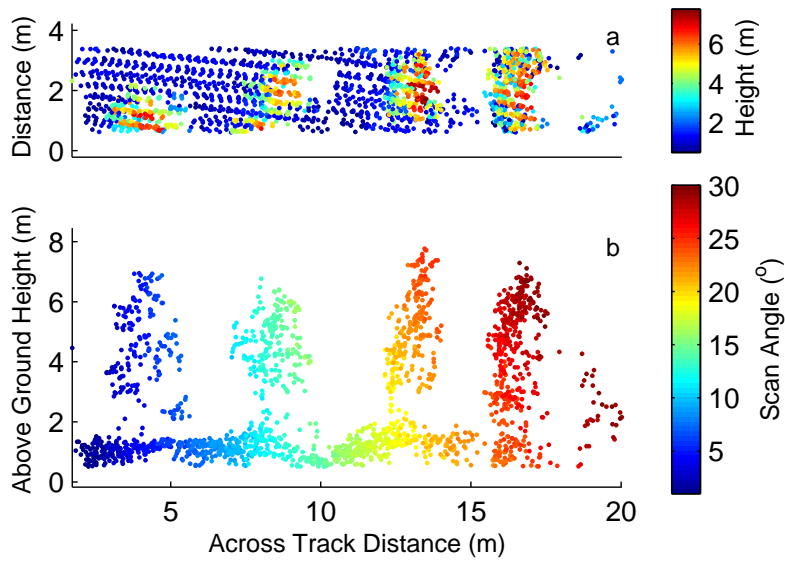

Figure 7. a) Top view and b) profile plot of a strip of a point cloud demonstrating the shadowing effect which increases as scan angle increases.

flying height is a significant attenuation of the number of returns received from the upper part of the canopy. It is evident that increased above ground flying heights result in a decreased return intensity from the top of the canopy which becomes insufficient to trigger a return at flying heights greater than $50 \mathrm{~m}$. As the Ibeo LUX scanner has a working range of $200 \mathrm{~m}$, the signal attenuation is most likely due to a combination of the canopy structure (i.e. small surface areas to trigger a return), the reflectance properties of the vegetation, the footprint size as well as the triggering mechanism of the Ibeo LUX laser scanner. Artefacts of this attenuation are still evident at flying heights below $50 \mathrm{~m}$. To determine if this small amount of attenuation effects the measurement of forest metrics, or if attenuation is occurring in all point clouds, comparison with field measurements of tree height and canopy properties (width and volume) is required.

Previous studies that have focused on the effect of point density on the measurement of forest structure have suggested that increased point densities result in an improved accuracy at the tree level (Disney et al., 2010; Lovell et al., 2005). It has also been shown that increased point density has no significant effect on metrics derived at the stand or plot level (Tesfamichael et al., 2010). In this study we found that decreased point density reduced the number of returns from the upper part of tree crowns. This is in line with the findings of Disney et al. (2010) and Lovell et al. (2005). However, it is also highly evident that a reduction in point density is not contributing to the attenuation of crown returns in the point clouds generated at higher flying heights. For point densities between 30 and 70 point per $m$ at the plot level all statistics were reliably derived. Furthermore, the collection of high density data, from a UAV platform, over a plot sized area does not incur a significantly higher cost. As such point density should not be a controlling factor in the design of a survey for multi-temporal studies.

The poor quality data collected from the higher altitudes (higher than $50 \mathrm{~m}$ ), limited the ability to resolve the effects of scan angle and footprint size within this study. Morsdorf et al. (2006) highlighted that increases in scan angle result in a reduction in canopy penetration due the distance the beam has to travel through the canopy and a reduction. This was also observed in the UAV generated point clouds as the shadowing effect shown in Figure 7 resulted in parts of the plot not being observed. These areas primarily consisted of the lower parts of canopy and some ground areas. This suggests that narrow scan angle ranges should be used where possible. No effect due to footprint size was observed in the data analysed. However, as previously discussed, it is highly likely that large footprint sizes are contributing to the attenuation of canopy returns with flying height.

Based on these results several recommendations for this specific UAV- LiDAR system and for UAV-borne LiDAR systems in general can be made. Primarily, this study shows that both flying heights above $50 \mathrm{~m}$ and the use of high scan angles should be avoided where possible. Although a different miniaturised laser scanner may perform better at higher altitudes, the potential for the use of UAVs is seen to be most beneficial at the plot level. As individual plots can be surveyed at flying heights below 50 $\mathrm{m}$ within a single flight, this recommendation holds for all UAV surveys at this scale. Furthermore, the spatial accuracy of the generated point clouds suggests the fusion of forward and backward transects is a feasible option to further restrict the scan angles used within lower altitude flights. In the case of this study, a $12.62 \mathrm{~m}$ radius circular plot is the primary area of interest. To completely cover this area a flying height of 20 mabove the tree height, would theoretically allow the entire plot to be covered with a maximum scan angle of $17.5^{\circ}$. Whereas this could be reduced to $12^{\circ}$ if the point clouds collected from forward and reverse transects were used to generate a single point cloud, while insuring $50 \%$ overlap.

This study has shown that with correctly chosen flying conditions,statistics which have been shown to be related to key forest metrics can be derived with high repeatability from UAVborne LiDAR systems. This suggests that any changes in forest structure, such as a loss of biomass due to disease or pruning, should be observable from multi-temporal surveys. One of the primary advantages of a UAV-system is an ability to fly ondemand. Therefore, the UAV can be used to assess a number of the dynamics which are important in modern forestry management. For example, an assessment of biomass loss due to disease could be tracked through time. Another advantage of UAV systems is their ability to generate very high resolution datasets. Although it has not been a focus of this study, the measurement of forest structure at the tree level is highly feasible with this platform and requires further investigation. Such analysis could aid studies into carbon modelling and the calibration of full scale data. Furthermore, the use of return intensity as an indicator of forest structure is becoming an important tool in forestry management (García et al., 2010). The use of a UAV-borne scanners limits the range and atmospheric effects in the measurement of intensity. This suggest that UAV-borne intensity metrics could be more accurate than similar full scale metrics.

In conclusion, the mapping of forest structure using the TerraLuma UAV-borne LiDAR system has been demonstrated to be feasible at the plot level. There are a number of potential uses for UAVborne LiDAR within forest management some of which will be the focus of the research with this system. However, the next stage of this research will focus on the comparison and calibration of the tree level metrics with ground inventory data.

\section{References}

Disney, M., Kalogirou, V., Lewis, P., Prieto-Blanco, a., Hancock, S. and Pfeifer, M., 2010. Simulating the impact of discrete-return lidar system and survey characteristics over young conifer and broadleaf forests. Remote Sens. Environ. 114(7), pp. 1546-1560.

Donoghue, D., Watt, P., Cox, N. and Wilson, J., 2007. Remote sensing of species mixtures in conifer plantations using LiDAR height and intensity data. Remote Sens. Environ. 110(4), pp. 509-522. 
García, M., Riaño, D., Chuvieco, E. and Danson, F. M., 2010. Estimating biomass carbon stocks for a Mediterranean forest in central Spain using LiDAR height and intensity data. Remote Sens. Environ. 114(4), pp. 816-830.

Goodwin, N. R., Coops, N. C. and Culvenor, D. S., 2006. Assessment of forest structure with airborne LiDAR and the effects of platform altitude. Remote Sens. Environ. 103(2), pp. 140-152.

Hopkinson, C., 2008. The uncertainty in conifer plantation growth prediction from multi-temporal lidar datasets. Remote Sens. Environ. 112(3), pp. 1168-1180.

Jaakkola, A., Hyyppä, J., Kukko, A., Yu, X., Kaartinen, H., Lehtomäki, M. and Lin, Y., 2010. A low-cost multi-sensoral mobile mapping system and its feasibility for tree measurements. ISPRS J. Photogramm. 65(6), pp. 514-522.

Lim, K. S. and Treitz, P. M., 2004. Estimation of above ground forest biomass from airborne discrete return laser scanner data using canopy-based quantile estimators. Scandinavian Journal of Forest Research 19(6), pp. 558-570.

Lovell, J., Jupp, D., Newnham, G., Coops, N. and Culvenor, D., 2005. Simulation study for finding optimal lidar acquisition parameters for forest height retrieval. Forest Ecology and Management 214(1-3), pp. 398-412.

Morsdorf, F., Kötz, B., Meier, E., Itten, K. and Allgöwer, B., 2006. Estimation of LAI and fractional cover from small footprint airborne laser scanning data based on gap fraction. Remote Sens. Environ. 104(1), pp. 50-61.

Næsset, E., 2009. Effects of different sensors, flying altitudes, and pulse repetition frequencies on forest canopy metrics and biophysical stand properties derived from small-footprint airborne laser data. Remote Sensing of Environment 113(1), pp. $148-159$.

Streutker, D. and Glenn, N., 2006. LiDAR measurement of sagebrush steppe vegetation heights. Remote Sens. Environ. 102(12), pp. 135-145.

Tesfamichael, S., Ahmed, F. and van Aardt, J., 2010. Investigating the impact of discrete-return lidar point density on estimations of mean and dominant plot-level tree height in Eucalyptus grandis plantations. Int. J. Remote Sens. 31(11), pp. 2925 2940 .

Vepakomma, U., Stonge, B. and Kneeshaw, D., 2008. Spatially explicit characterization of boreal forest gap dynamics using multi-temporal lidar data. Remote Sens. Environ. 112(5), pp. 2326-2340.

Wallace, L., Lucieer, A., Turner, D. and Watson, C., 2011. Error assessment and mitigation for hyper-temporal UAV-borne LiDAR surveys of forest inventory. In: SilviLaser, Hobart, Tasmania.

Woods, M., Pitt, D., Penner, M., Lim, K., Nesbitt, D., Etheridge, D. and Treitz, P., 2011. Operational implementation of a lidar inventory in boreal ontario. The Forestry Chronicle 87(04), pp. 512-528.

Yu, X., Hyyppä, J., Vastaranta, M., Holopainen, M. and Viitala, R., 2011. Predicting individual tree attributes from airborne laser point clouds based on the random forests technique. ISPRS J. Photogramm. 66(1), pp. $28-37$.

\section{ACKNOWLEDGEMENTS}

We acknowledge the Winfred Violet Scott Trust for funding support in purchasing the OktoKopter and the laser scanner. The Australian Antarctic Division are acknowledge for providing workshop time in constructing the sensor mount. We also acknowledge the considerable help and technical know how of Tony Veness in design and construction of the sensor mount. Thanks to Darren Turner for his input and UAV piloting skills. 